\title{
Observation of slow down of polystyrene nanogels diffusivities in contact with swollen polystyrene brushes
}

\author{
V.N. Michailidou ${ }^{1,3}$, B. Loppinet ${ }^{1, a}$, C.D. Vo ${ }^{2, b}$, J. Rühe ${ }^{2}$, K. Tauer ${ }^{4}$, and G. Fytas ${ }^{1,3}$ \\ 1 FORTH/Institute of Electronic Structure and Laser, Heraklion, Greece \\ 2 Department of Microsystems Engineering, IMTEK, University of Freiburg, Freiburg, Germany \\ 3 Department of Materials Science and Technology, University of Crete, Heraklion, Greece \\ 4 Max Plank Institute for Colloid and Interface Science, Golm, Germany
}

Received 9 November 2007 and Received in final form 22 February 2008

Published online: 13 May 2008 - (C) EDP Sciences / Società Italiana di Fisica / Springer-Verlag 2008

\begin{abstract}
The diffusion of dilute colloids in contact with swollen polymer brushes has been studied by evanescent wave dynamic light scattering. Two polystyrene nanogels with $16 \mathrm{~nm}$ and $42 \mathrm{~nm}$ radius were put into contact with three polystyrene brushes with varying grafting densities. Partial penetration of the nanogels within the brushes was revealed by the evanescent wave penetration depth-dependent scattering intensities. The experimental short-time diffusion coefficients of the penetrating particles were measured and found to strongly slow down as the nanoparticles get deeper into the brushes. The slow down is much more marked for the smaller $(16 \mathrm{~nm})$ nanogels, suggesting a size exclusion type of mechanism and the existence of a characteristic length scale present in the outer part of the brush.
\end{abstract}

PACS. 61.25.he Polymer solutions - 47.57.-s Complex fluids and colloidal systems

\section{Introduction}

Recent developments in the area of microfluidics have fostered the regain of interest into the longstanding problem of flow and diffusion close to surfaces $[1,2]$ as the movement of particles close to functionalized surfaces is of importance in many practical devices. The case of the hard wall is one special case that has received significant attention and the boundary conditions are now well established and understood. However, more complex situations may arise for example in the case of polymer-decorated surfaces, like the highly desired slippery boundaries that increase the near wall flow velocity [1]. On the opposite side, sticky surfaces may also be desirable in some cases in order to attract particles or to reduce surface slip, like in the case of polymer melt flow, where surface-grafted polymer chains are used to reduce the slip of flowing polymer melts [3].

Grafting polymer chains [4] constitute a popular way to modify surfaces. Polymer brushes refer to ensembles of end-grafted macromolecules attached to a solid surface with high enough grafting densities so that the polymeric coils adopt a stretched conformation perpendicular to the surface. Their structure is governed by two main

\footnotetext{
a e-mail: benoit@iesl.forth.gr

b Present address: School of Pharmacy and Pharmaceutical Sciences, University of Manchester, UK.
}

parameters, the molecular weight and the grafting density as they determine the segment density profile of the polymer layer close to the surface. The surface-attached chains modify the interaction of particles present in a contacting solution. Large enough particle will not penetrate the brush and a theoreteical expression for the force exerted by the brush on such large hard sphere exists [5]. For smaller particles, organization by the polymer brushes has recently been theoretically described [6] and the authors predicted that polymer-soluble particles smaller than a brush-determined threshold should penetrate in the brush up to a given depth that scales inversely with particle volume. Non-hard surfaces and their effects on particles diffusion have also been considered [7].

In recent experimental work [8], we observed that the permeability of swollen polymer brushes to colloidal particles depended on the particles size and nature and that the diffusivities of the particles in the vicinity of the brush were influenced by the brush presence: Whereas large hard spheres were found to not penetrate the brush and exhibited solution like diffusivities with a certain drag reduction from the brush, smaller and softer particles were found to partially penetrate the brush and to exhibit slower diffusivities reflecting the increased friction within the brush.

In this work, we further investigate the diffusivities of colloidal particles within swollen brushes, extending the study on the effect of the grafting density of the brushes on both the particles penetration depth and 
their diffusivities. Three polystyrene brushes with similar molecular weight and different grafting densities are used and brought into contact with dilute dispersions of two polystyrene nanogels. The experimental conditions covered the accessible conditions within the used chemistry. Evanescent wave dynamic light scattering is employed to measure both scattered intensities and short-time diffusivity of the nanoparticles in and close to the polymer brush. Partial penetration of polystyrene nanogels within the brush layer is reported as well as slow diffusivities in the layer.

\section{Experimental}

\section{Polymer brushes}

The polystyrene brushes were prepared by surfaceinitiated polymerization using a glass surface as a substrate. The chains were grown via free radical polymerization from an azo initiator previously covalently attached to the glass surface [9]. Molecular weight and grafting density are varied by controlling the polymerization time and the monomer concentration and details about the molecular properties of these brushes are described elsewhere [10]. Three brushes were used with an averaged distance between grafting site of 3,5 and $8 \mathrm{~nm}$ for the PS3, PS5 and PS8 samples, respectively, and molecular weight of $10^{6} \mathrm{~g} / \mathrm{mol}$. As a result of the rather large expected polydispersity of the free radical polymerization, the polymer concentration profile is expected to be broader than the parabolic profile of monodisperse brushes [9-11]. When swollen in a good solvent, such PS brushes with large molecular weight chains can reach thicknesses of the order of $1 \mu \mathrm{m}[9,10]$.

\section{Polystyrenes nanogels}

Two nanogel polystyrene particles were used with different particle size $\left(R_{\mathrm{h}}=16 \mathrm{~nm}\right.$ and $\left.R_{\mathrm{h}}=42 \mathrm{~nm}\right)$. The nanogel samples were prepared by aqueous heterophase polymerizations with specially adopted recipes in order to get these small-sized and cross-linked particles. For the $16 \mathrm{~nm}$ spheres $2 \mathrm{~g}$ of styrene together with $0.076 \mathrm{~g}$ of $1,3-$ diisopropylene benzene as cross-linker and $0.05 \mathrm{~g}$ of 2,2 azobisisobutyronitrile as initiator were dispersed in $94 \mathrm{~g}$ of water containing $4 \mathrm{~g}$ of cetyltrimethylammonium bromide as stabilizer for several hours at room temperature with magnetic stirring in a glass reactor equipped with condenser under nitrogen atmosphere. Then the mixture was heated to $65^{\circ} \mathrm{C}$ for $12 \mathrm{~h}$ hours. The larger spheres $(R=42 \mathrm{~nm})$ were prepared by polymerizing $10 \mathrm{~g}$ of styrene together with $0.38 \mathrm{~g}$ of 1,3-diisopropylene benzene, $0.5 \mathrm{~g}$ of sodium dodecylsulfate as stabilizer, and $0.32 \mathrm{~g}$ of potassium peroxodisulfate as initiator at $80^{\circ} \mathrm{C}$ for 3 hours under nitrogen atmosphere. The solids of the latexes were isolated by freeze drying. Auxiliary materials were removed by repeated washing of the solids with hot methanol and precipitation from a solution in tetrahydrofuran with methanol. Finally, the solids were dried 48 hours at $50^{\circ} \mathrm{C}$ in a vacuum oven.

Beside the hydrodynamic radius $R_{\mathrm{h}}$ measured by dynamic light scattering, the weight averaged molecular weight $M_{\mathrm{w}}$, and the gyration radius $R_{\mathrm{g}}$ have been obtained from static scattering measurements of dilute toluene solutions, The mass and gyration radius are $M_{\mathrm{w}}\left(10^{6} \mathrm{~g} / \mathrm{mol}\right)=$ $2.6 / 25 R_{\mathrm{g}}(\mathrm{nm})=14 / 38$ for the $16 / 42 \mathrm{~nm}$ hydrodynamic radius particles. The relatively high crosslink density leads to a system, which is only weakly swollen even in good solvents for the polymer. The average concentration within the nanogel can be calculated according to $c=M_{\mathrm{w}} /\left[(4 / 3) \pi R_{\mathrm{h}}{ }^{3}\right]$ and is on the order of $0.15 \mathrm{~g} / \mathrm{cm}^{3}$ for both nanogels. The relatively high polymer concentration within the nanogel particles is expected to lead to a hardsphere-like behavior, as in the case of larger PS microgels used as model hard spheres [12].

\section{Evanescent wave dynamic light scattering}

Evanescent wave dynamic light scattering (EWDLS) is a variant of dynamic light scattering which uses a total internal reflection configuration in order to probe specifically the solid-liquid interface [13-19]. When a light beam impinges an interface between two dielectric media with an angle of incidence larger than the critical angle of internal reflection, an evanescent field $E_{\text {ev }}$ penetrates the medium of lower refractive index over a distance of the order of the wavelength. The field at a distance $z$ from the interface is

$$
E_{\mathrm{ev}}=E_{0}(r) \exp (-\kappa z)
$$

where $E_{0}$ is the amplitude of the incident field, and $\kappa^{-1}$ is the penetration depth. The presence of a scatterer at position $z$ gives rise to a scattered intensity proportional to the evanescent intensity $\left|E_{\mathrm{ev}}(z)\right|^{2}$. In the simplest case of uncorrelated scatterers, the total averaged intensity measured at a scattering wave vector $\boldsymbol{q}$ is simply the addition of the contribution of individual scatterers present in the illuminated volume. In the case of large enough number of particles, the sum can be replaced with an integral

$$
I_{s}(q)=\sum\left|E_{\mathrm{ev} i}\right|^{2} \cong \int_{z}\left|E_{\mathrm{ev}}\right|^{2}(z) n(z) P(q) \mathrm{d} z,
$$

where $n(z)$ is the number density profile of the particles along the $z$-direction and $P(q)$ is the form factor of particles. $n(z)=c(z) S$, where $c(z)$ is the local concentration and $S$ is the beam cross-section. The penetration depth dependence of the scattered intensity at constant $q$ is directly related to the concentration profile, as

$$
I_{s}(q, \kappa) \approx I_{0}(q) \int_{z} n(z) \exp (-2 \kappa z) \mathrm{d} z .
$$

The concentration profile $n(z)$ arises as a result from the interaction between the surface and the scatterers. If $V(z)$ is the interaction potential between the interface and 


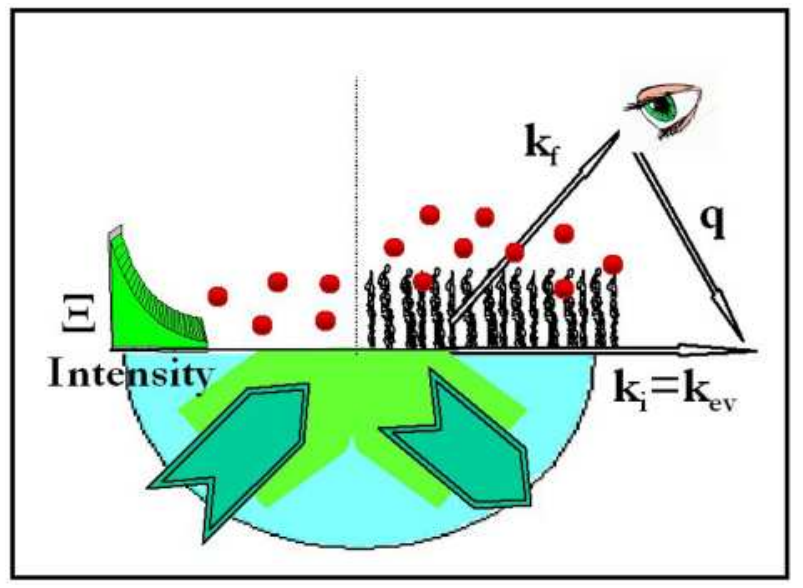

Fig. 1. (Colour on-line) Schematic diagram of the evanescent wave dynamic light scattering set-up with the laser beam undergoing a total reflection and the evanescent field penetrating within a distance $\Xi$ from the surface. The wave vectors $\boldsymbol{k}_{\text {ev }}$ and $\boldsymbol{k}_{f}$ refer to the incident and scattered beams, with $\boldsymbol{q}$ being the scattering wave vector.

a particle at distance $z$, a Boltzmann distribution is expected for $n(z)$,

$$
n(z)=n_{0} \exp [-V(z) / k T]
$$

where $n_{0}$ is the concentration far from the wall and $k T$ is the thermal energy.

The concentration profile of scatterers perpendicular to the interface $n(z)$ is related to the penetration dependence of the scattered intensity, by an inverse laplace transform $[12,16]$. The measure by EWDLS of the penetration depth dependence of the scattered intensity can provide useful information about $n(z)$, i.e. the spatial distribution of particles in the case of dilute solutions of particles.

Besides the often overlooked intensity information, EWDLS provides information on the Brownian dynamics of the particles, through the computed intensity autocorrelation function. The relative motion of the particles within the scattering volume gives rise to time fluctuation of the scattered intensity, as in standard DLS experiments, but, due to the exponentially decaying evanescent intensity away from the glass, the particles closer to the glass are weighted more.

The used experimental set-up was similar to the one described in previous work $[8,10]$ and is schematically depicted in Figure 1. The evanescent light was generated by total internal reflection of the incident laser $(\lambda=532 \mathrm{~nm})$ beam at the interface of a brush grafted semi-cylindrical lens. A cylindrical solvent cell with the semi-cylindrical lens in the centre was placed in a precision $\theta-2 \theta$ goniometer. The independent rotation of the cell and of a singlemode optical fiber detection permits to control both the scattering angle $\theta$ and the incidence angle $\theta_{1}$, that govern, respectively, the value of the scattering wave vector $q$ and the penetration depth $\kappa^{-1} \cdot \kappa^{-1}=k_{n}^{-1} /\left(\sin ^{2} \theta_{1}-\right.$ $\left.\sin ^{2} \theta_{c}\right)^{1 / 2}$, with $\theta_{c}$ being the critical angle of the total internal reflection and $k_{n}$ the wave vector of light in the solvent of the refractive index $n, k_{n}=2 \pi n / \lambda$. The scattering wave vector is defined as $\mathbf{q}=\mathbf{k}_{f}-\mathbf{k}_{\mathrm{ev}}$, with $\mathbf{k}_{\mathrm{ev}}$ and $\mathbf{k}_{f}$ being the wave vectors of the evanescent and scattered light. The magnitude of the scattering wave vector $q$ is $q \cong(4 \pi n / \lambda) \sin (\theta / 2)$, where $\theta$ is the scattering angle since $q / \kappa>1$ in all the experiments. All measurements were performed at room temperature. The scattered intensity autocorrelation function $g(q, \kappa, t)$ intensity was recorded over a broad time range by the ALV-5000 fast digital correlator. The strong elastic scattering from the rough glass substrate provides a heterodyning source for the fluctuating intensity of interest. The normalized correlation obtained as

$$
C(q, \kappa, t)=\frac{g(q, \kappa, t)}{g\left(q, \kappa, t_{\rightarrow 0}\right)}
$$

is equal to the normalised field-autocorrelation function [20]. In heterodyne conditions, the dynamic scattered intensity arising from the particle/brush system is deduced as

$$
\langle I\rangle(q, \kappa)=I_{0} \cdot g(q, \kappa, t \rightarrow 0)
$$

where $I_{0}$ is the averaged intensity over the measurement time. This dynamic scattering $\langle I\rangle(q, \kappa)$ is dominated by the large particles (compared to the brush) and is therefore solely attributed to the nanogel particles.

In the absence of any aggregation and/or other interparticle interaction phenomena, the motion of individual particles is probed and the measured correlation functions are directly related to the single-particle diffusion. However, due to the total internal reflection configuration and the associated strong decay of the evanescent wave, the relation between $C(q, t)$ and the mean-square displacement is not as straightforward as the classical 3D diffusion case. The normalized $C\left(q, \kappa^{-1}, t\right)$ were analyzed using a first cumulant type of approach, where an early decay rate was deduced from the initial slope of $\log [C(q, t)]$, $\log [C(q, t)]=-\Gamma t+O\left(t^{2}\right)$. The early linear part used for determining $\Gamma$ was found to typically extend over a region from 1 to 0.8 (see Figs. 3 and 4 below).

It was recently shown that the effective diffusivity $\langle D\rangle=\frac{\Gamma}{\left(q^{2}+\kappa^{-2}\right)}$ is related to the $z$-dependence of the diffusivity $D(z)$ in the case of isotropic diffusion considered here, as [18]

$$
\langle D\rangle=\int D(z) \exp (-2 \kappa z) \mathrm{d} z
$$

The penetration depth dependence of the apparent diffusivity provides a measure of the Laplace transform of the $z$-dependent mobility of the particles; this relation is similar to the relation between the penetration depth dependence of the scattered intensity and the concentration distribution of the scatterers (Eq. (3)). 
a)

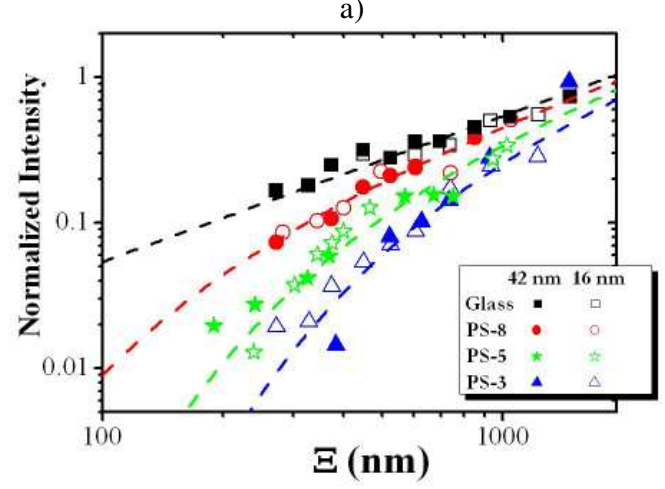

b)
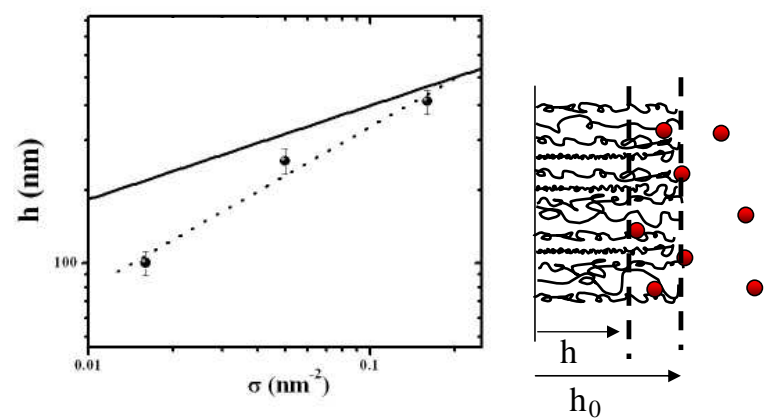

Fig. 2. (Colour on-line) a) Intensity $I(\Xi)$ profile for the PS nanogel particles in the case of a solid surface $(\boldsymbol{\square})$ and in contact with the three brushes; PS-3 ( $\mathbf{\Delta})$, PS-5 $(\star)$ and PS-8 $(\bullet)$ for the larger (solid symbols) and the smaller particles (open symbols). The lines are the best fit of step concentration profiles (see text). b) Excluded heights $h$ obtained from the fit in a) as a function of the brush grafting density. The line is the expected scaling for brush height $h_{0}$.

\section{Results and discussion}

\section{A: Brush penetrability}

As discussed in the previous section, the distribution of the particles normal to the glass wall can be estimated from the evanescent wave penetration depth dependence of the evaluated scattered intensity. Figure 2 displays the reduced scattered intensities obtained for a varying penetration depth at a constant $q=0.02 \mathrm{~nm}^{-1}$ (corresponding to $90^{\circ}$ scattering angle) for the two microgels suspended in toluene, in contact with the three swollen brushes as well as a bare glass prism. Remarkably, the experimental data obtained for the two nanogels in contact with a given brush are almost superimposed for all three brushes. It suggests that within the explored particle size range and the technique's resolution, the brush penetrability is controlled by the brush rather than the particle radius. The lines in Figure 2 represent the best fits of the experimental points obtained for a hypothetical step-like concentration profile, i.e. $z>h, n(z)=n_{0}$ and $z<h, n(z)=0$, corresponding to a hard-core/excluded-volume potential

$$
I(\kappa)=\frac{1}{2} I_{0} \kappa^{-1} n_{0} \exp (-2 h \kappa) .
$$

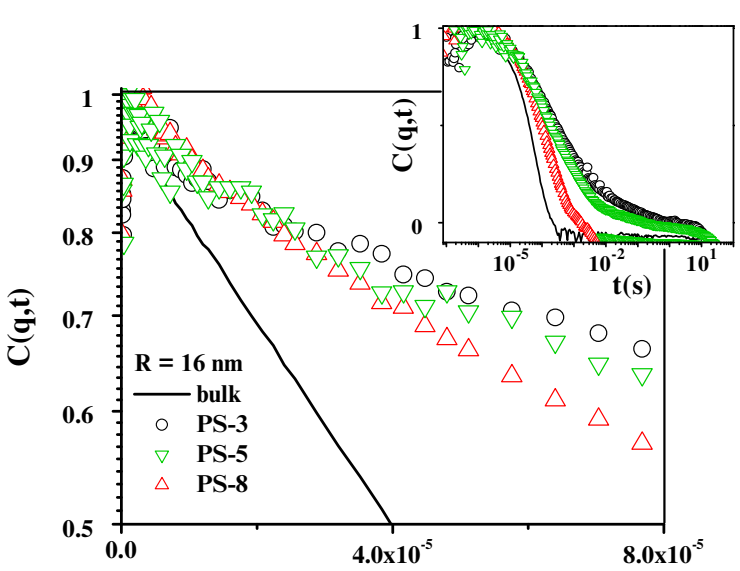

$\mathbf{t}(\mathbf{s})$

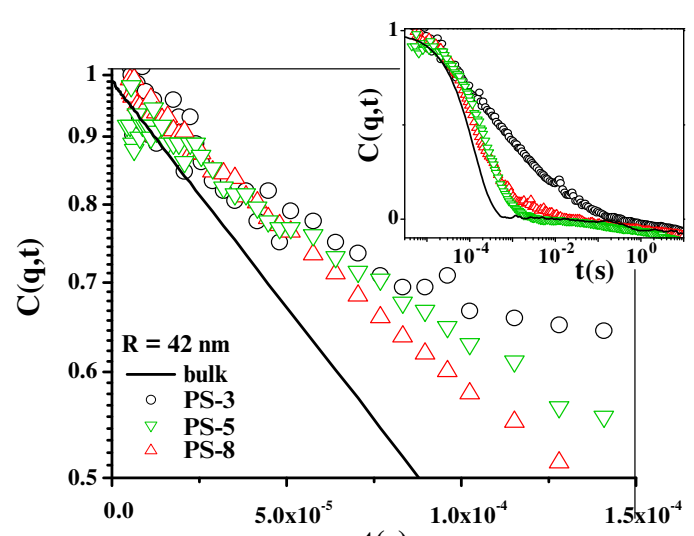

$\mathbf{t}(\mathbf{s})$

Fig. 3. (Colour on-line) Normalized intermediate scattering functions $C(q, t)$ of the PS nanogels in contact with the 3 brushes at the same $q\left(q=0.02 \mathrm{~nm}^{-1}\right)$ and the same large penetration depth $(\Xi=900 \mathrm{~nm})$ in toluene at room temperature with top: dilute solutions of $42 \mathrm{~nm}$ and bottom: $16 \mathrm{~nm}$ nanogel particles. Inset: the same full correlation functions on a wider time range presented on a logarithmic scale.

Clearly the agreement with this simple profile is rather satisfactory. The obtained values for the excluded layer thickness $h$, which is a measure of how close to the glass surface the particle can travel, are $L_{\mathrm{PS}-3}=375 \pm 30 \mathrm{~nm}$, $L_{\mathrm{PS}-5}=230 \pm 25 \mathrm{~nm}$ and $L_{\mathrm{PS}-8}=100 \pm 10 \mathrm{~nm}$. These values demonstrate the overall exclusion of the particles with $2 R_{1}=84 \mathrm{~nm}$ and $2 R_{2}=32 \mathrm{~nm}$ from the brushes. The same brushes were found to present an average dynamic mesh sizes $3 \leq \xi \leq 8 \mathrm{~nm}$ [3], and exclusion of particles with radius of the order of $10 \times \xi$ from the brush might be expected [21].

When reported as a function of the grafting density (Fig. 2b), the deduced $h$ values are expectedly found to increase with the grafting density. Assuming a power law dependence, we obtain $h \sim \sigma^{0.57}$. This exponent is clearly larger than the theoretical scaling exponent for the brush thickness $\left(h_{0} \sim \sigma^{1 / 3}\right.$ within the Alexander-DeGennes model) [21]. With increasing the brush grafting density the particles are kept at a further and further distance from the glass surface so that the volume inaccessible to the 

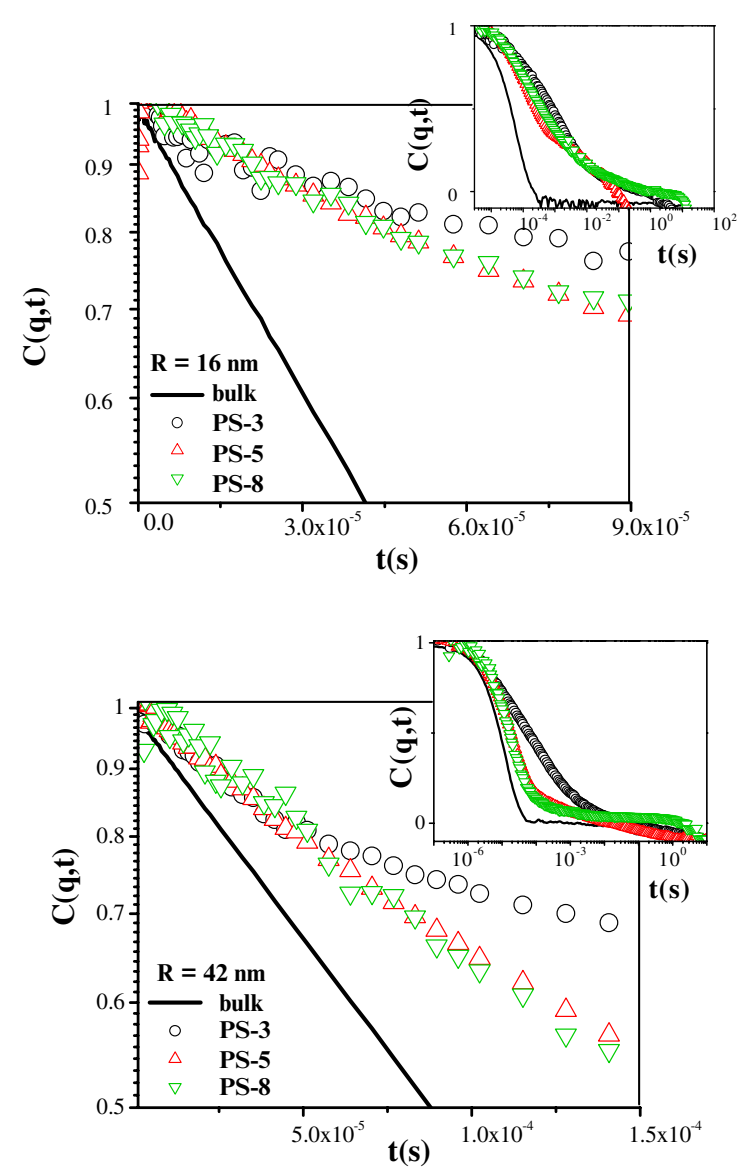

Fig. 4. (Colour on-line) Normalized intermediate scattering functions $C(q, t)$ of the PS nanogels in contact with the 3 brushes at the same $q\left(q=0.02 \mathrm{~nm}^{-1}\right)$ and the same large penetration depth $(\Xi=700 \mathrm{~nm})$ in toluene at room temperature with top: dilute solutions of $42 \mathrm{~nm}$ and bottom: $16 \mathrm{~nm}$ nanogel particles. Inset: The same full correlation functions on a wider time range presented on a logarithmic scale.

particles increases faster than the expected brush height, and the exclusion becomes stricter. Within the technique resolution, the extent of the visited region in a given brush appears to be similar for both nanogels (16 and $42 \mathrm{~nm}$ ).

\section{B: Particle diffusion}

We now turn to the diffusion of the nanoparticles in the vicinity of the polymer brushes. Normalized correlation functions measured for different particle-brush systems at a given wave vector $\left(q=0.02 \mathrm{~nm}^{-1}\right)$ are reported in Figures 3 and 4 both in $C$ vs. $\log$ (time) (inset) and $\log C$ vs. time representations highlighting, respectively, the overall decay over the full time scale and the early part of the decay used to determine the fast relaxation rate $\Gamma$.

Figure 3 presents the normalized correlation functions measured at the largest $900 \mathrm{~nm}$ evanescent wave penetration depth for the smaller $16 \mathrm{~nm}$ nanogels (top) and the larger $42 \mathrm{~nm}$ ones (bottom). The different symbols correspond to the different brushes, and the solid line to the

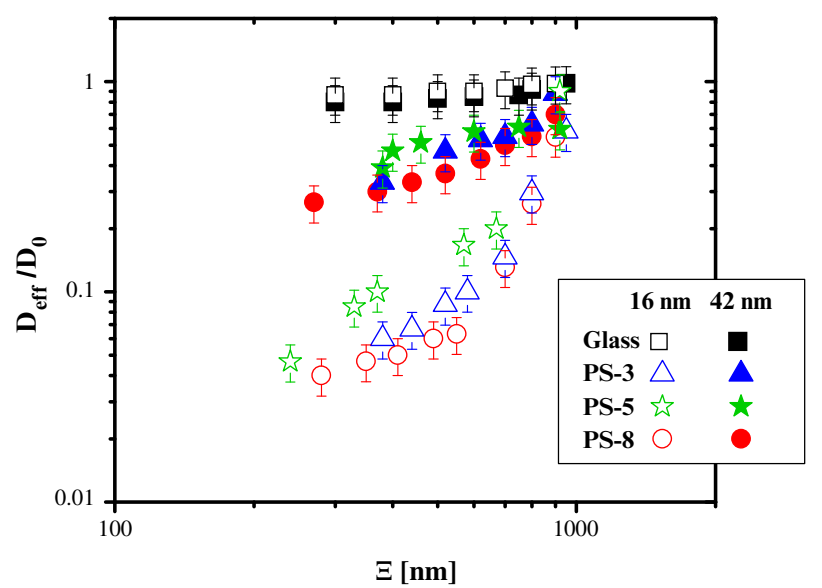

Fig. 5. (Colour on-line) Normalized apparent diffusivities $D_{\text {eff }} / D_{0}$ ratio where $D_{0}$ is the diffusion coefficient of the bulk vs. penetration depth $\Xi$. The solid surface is represented by

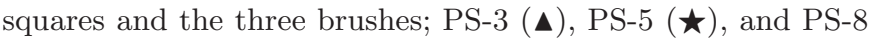
$(\bullet)$. Larger $42 \mathrm{~nm}$ nanogels are represented by solid symbols and smaller $16 \mathrm{~nm}$ nanogels by open symbols.

bulk nanogel solution. Figure 4 presents in a same way the data at a shorter $700 \mathrm{~nm}$ penetration depth. In order to allow easy comparisons, the same scaling has been adopted for the four cases, in the main figures the correlation scale is from 1 to 0.5 and the time scale is from 0 to $2 \ln 2 / \Gamma_{0}$, where $\Gamma_{0}$ is the bulk decay rate. The decays measured for the nanogels in contact with the brushes (points) are clearly slower than the decays of the bulk solution (lines), revealing the slow down of the nanogels dynamics at contact with the brushes. Evaluating the different brush/nanogel cases, a comparison of top and bottom graphs of Figures 3 and 4 reveals that the smaller $16 \mathrm{~nm}$ particles experience a stronger slow down than the larger $42 \mathrm{~nm}$ ones at both penetration depths. Similarly, a comparison between Figure 3 and 4 shows that for a given nanogel the decays are slower for the shorter $700 \mathrm{~nm}$ penetration depth than for the larger $900 \mathrm{~nm}$ one. Finally, we note that in each graph the decays of the three different symbols (corresponding to the same nanogel at the three different brushes) almost superimpose in the early part (correlation from 1 down to 0.8). Therefore, the early decay rates for a given nanogel in contact with the three different brushes are very similar.

The slow and broad decays observed in the presence of the brushes clearly reflect the slower particles diffusion due to the presence of polymeric environment and can be taken as a confirmation of some level of interaction/interpenetration of the particles within the brushes, as inferred from the penetration depth dependence of the scattered intensities (Fig. 2).

The early decay rates $\Gamma$ obtained from an initial slope analysis are transformed into the apparent short-time diffusivities defined as $D_{\text {eff }}\left(\kappa^{-1}\right)=\frac{\Gamma}{q^{2}+\kappa^{-2}}$. Figure 5 reports the short-time diffusion coefficient normalized by the free bulk solution diffusion coefficient $D_{0}$ as a function of the evanescent wave penetration depth $\Xi=\kappa^{-1}$ for the different brush/nanogel systems, as well as for the 
nanogels/bare glass systems (Fig. 1). The normalized apparent fast diffusion coefficients are expectedly found to increase with the evanescent wave penetration depth in all cases and to approach 1 at larger $\Xi$ The data in Figure 5 fall into three groups: the bare glasses for the two nanogels (full and empty squares) present the weakest $\Xi$ dependence, the large $42 \mathrm{~nm}$ nanogel at the three brushes (other full symbols) fall together with an intermediate $\Xi$ dependence and the small $16 \mathrm{~nm}$ nanogel at the three brushes (other empty symbols) present the strongest $\Xi$ dependence. The insensitivity of $D_{\text {eff }} / D_{0}$ to the brush grafting density confirms the earlier observation of the similar early decays in Figures 3 and 4.

The $\Xi$ dependence is a consequence of the slowing down of the particles as they move closer to the glass surface and reflects a strong $z$ dependence of $D$, much stronger than the hydrodynamic slow down at a solid surface. In the hard-surface case, the observed slow down is known to be the result of the hydrodynamic slow down induced by the presence of the hard wall $[1,2,18,22]$. The polymer has a stronger effect on the nanogels mobility than the hard wall. The $\Xi$ dependence is much more marked for the smaller $16 \mathrm{~nm}$ nanogels than for the larger $42 \mathrm{~nm}$ particles. At the shorter penetration depth, the $D_{\text {eff }}$ is down to $D_{0} / 25$ compared to $D_{0} / 2$.

Beside the fast diffusivities quantified in Figure 5, the measured correlation functions of Figures 3 and 4 also provide a qualitative picture of the long-time behavior. Indeed the stronger slow down of the short-time diffusion also leads to the broader long-time decay of the correlation function. Upon closer inspection of the correlation functions, the departures from the linear regime appear more marked for the denser brush and reveal systematic differences between the three brushes. The tendency is more evident for the $42 \mathrm{~nm}$ nanogels, but also present in the $16 \mathrm{~nm}$ ones. These deviations could be interpreted as arising from a strong gradient of diffusion coefficient $D(z)[18] \mathrm{b},[22]$. In extreme cases, correlation functions spanning four decades of time can be observed for the $16 \mathrm{~nm}$ particles in contact with the denser brush, whereas the $C(q, t)$ decay remains narrow for the $42 \mathrm{~nm}$ particles in contact with the same brush. Since the decay reflects the self-diffusion of the particles over characteristic distances of $1 /\left(q^{2}+k^{-2}\right)$, the very broad decays indicate that this motion at the brush contact can be slowed down up to 4 orders of magnitude compared to the diffusion in solvent. This extremely large slow down of the particles has to be envisaged as a trapping of the particles by the brush.

\section{Discussion}

The experimental results obtained for the spatial distribution of the nanogels within the brushes-decorated interfaces (Fig. 2) and the nanogels short-time diffusivities (Fig. 5) can be summarized as follows: the brushes are not fully penetrable to the particles, and the particles diffusivities are strongly affected by the brush.

These observations can be rationalized within the following scenario. Dense polymer brushes remain partially impenetrable to the nanogels, with a region in the outer part of the brush where the nanogels can penetrate and "feel" the polymer brush and experience an increased friction leading to the observed slower diffusion as schematically depicted in the inset of Figure 2b. The partial impenetrability of the dense brushes to the nanogels is corroborated by the findings that these same nanogels phaseseparate when dispersed in large molecular weight PStoluene solutions. In complementary experiments mimicking the brush conditions, a few drops of dilute solution of nanogels were added to a 10 weight $\%$ solution of $10^{6} \mathrm{~g} / \mathrm{mol} \mathrm{PS}$ in toluene. After homogenization, the nanogels were observed to slowly segregate from the polymer solution, forming macroscopic domains over very large time scale (days to weeks).

Within the outer interaction region of the brushes, the nanogels diffusion is significantly slower than the diffusion in the pure solvent, and more so as the nanogels get deeper into the brush, as the $z$-dependence of the diffusion coefficient is at the origin of the observed $\Xi$ dependence of the $D_{\text {eff }}($ Eq. (7)) (Fig. 5). The slow diffusion has to be attributed to an increased interaction/friction of the nanogels in the brush environment. It is worth noting that the case of the diffusion of colloidal particles in solutions and gels of non-adsorbing polymers has received much attention from theory, experiments and simulations [23]. Many possibilities have been considered but despite the large efforts the diffusion of colloids in such environment is still not fully understood. It is generally admitted that the experienced friction should lie between the solvent and the overall polymer solution viscosities [23].

The striking point in Figure 5 is that the brush effect on the diffusivities is much more pronounced for the smaller $16 \mathrm{~nm}$ nanogels than the larger $42 \mathrm{~nm}$ nanogels. This is somewhat counterintuitive since larger tracer particles are usually expected to be more affected than smaller particles, as for example in the case of semi-dilute polymer solutions with colloids tracer dispersed into it. A noticeable exception is the case of gel permeation chromatography (GPC), were larger polymer chains are eluted faster than shorter ones [24]. This is generally believed to arise from the "tortuosity" of the used gels, so that the smaller chains can explore a larger volume of pores and have a slower overall velocity, leading to the size exclusion mechanism. The diffusivities of the two nanogels in the vicinity of the polymer brush may also be influenced by other parameters beside their size, like crosslinking density or surface charge. They could be different in the two systems. In that respect, we note that both nanogels present (as already mentioned in the experimental part) similar swelling ratio $M / R_{h}^{3}$ and $R_{g} / R_{h}$, i.e. if they have a different internal structure, they lead to similar swelling. We also note that both nanogels present a similar moderate slowdown close to the glass hard wall (square in Fig. 5). When in contact with the glass surface, the surface chemistry does not induce large differences in diffusivities. Finally, attractive interaction between the polymer brush and the nanogels should lead to a nanogel enriched region in the vicinity of the brush, (compare to 
the bullk solution) and consequently a reverse curvature of the $\Xi$ dependence of the scattered intensity $I(\Xi)$ compared to the one observed in Figure 2. Therefore we favor the size difference as the primary origin of the different diffusivities within the brush.

If only the nanoparticles size is involved, the very strong slow down experienced by the $16 \mathrm{~nm}$ nanogels, and not present to the same extent in the larger $42 \mathrm{~nm}$ ones, will have to be explained by some commensurate topological interaction within an outer region of the brush. This would imply the presence of a specific length scale in the outer part of the three brushes. Further experiments with nanoparticles of similar sizes and different chemistry should help to corroborate one or the other possibility, i.e. the friction or the interaction origin of the slow down.

Due to the chain stretching in the direction perpendicular to the surface, we may expect an anisotropic experienced friction, which will result in a motion with different diffusion coefficients in plane and in the perpendicular direction. Such anisotropy has been evidenced in the case of flat hard surfaces. With the used set-up, the anisotropy of the diffusion can not unambiguously be resolved, however we did not observe any angular dependence that will imply a large anisotropic motion.

\section{Concluding remarks}

Using EWDLS and the penetration dependence of the scattered intensity and the early decay rate, we have observed the partial exclusion of PS nanogels from swollen PS brushes and the nanogel slowdown as they penetrated the polymeric environment, using three different brushes with different grafting densities in combination with two different colloidal nanogels. The measured dynamics clearly uncovered some large differences in the motion of the particles when in contact with the swollen brushes, where the larger particles are less trapped. The role of the nature of the particles remains to be resolved, and could be of importance. On the practical side, these findings suggest that polymer brush could be used for size exclusion purposes. EWDLS appears as a powerful technique to study the diffusivities of small particles near soft interfaces [7] that will not be feasible by the more standard optical microscopy [25].

The simple case of non-interacting particles in contact with polymer brushes reveals a rich dynamic behavior, form lubricating condition for fully non-penetrating hard sphsere [8] to the almost trapped smaller nanogels reported in this paper. Support of theory of the type of [5-7] as well as of computer simulations will be needed to shed more light on the swollen brush/particle specificities and to uncover the penetration of non-adsorbing particles within a polymer brush. Such progresses will allow the quantification of the observed phenomena and permit to identify whether their origin is purely entropic or required specific interactions between the brush and the particles.

\section{References}

1. L. Bocquet, J.L. Barrat, Soft Matter 3, 685 (2007).

2. E. Lauga, M.P. Brenner, H.A. Stone, Condens. Matter 501557 (2005).

3. L.A. Archer, Wall slip: measurement and modeling issues, in Polymer Processing Instabilities, edited by S.G. Hadzikiriakos, K.B. Migler (Marcel Dekker, New York, 2005), Chapter 4, p. 73.

4. R.C. Advincula, W.J. Brittain, K.C. Caster, J. Rühe (Editors), Polymer brushes (Wiley VCH, Weinheim, 2004); W.J. Brittain, S. Minko, J. Polym. Sci. A: Polym. Chem. 45, 3505 (2007); S.T. Milner, Science 251, 905 (1991); G. Grest, Adv. Polym. Sci. 138, 149 (1998).

5. J.U. Kim, M.W. Matsen, Macromolecules 41, 246 (2008).

6. B.O. O'Shaughnessy, J.U. Kim, Phys. Rev. Lett. 89, 238301 (2002); B.O. O'Shaughnessy, J.U. Kim, Macromolecules 39, 413 (2006).

7. T. Bickel, Phys. Rev. E 75, 041403 (2007).

8. E. Filippidi, V. Michailidou, B. Loppinet et al., Langmuir 23, 5139 (2007).

9. J. Rühe, O. Prücker, Langmuir 14, 6893 (1998).

10. V. Michailidou, B. Loppinet, O. Prücker et al., Macromolecules 38, 8960 (2005); V. Michailidou, B. Loppinet, O. Prücker et al., J. Polym. Sci. B: Polym. Phys. 44, 590 (2006).

11. M.E. Cates, S.T. Milner, T.A. Wilner, Macromolecules 21, 2160 (1988).

12. D. Sornette D., K.H. Lan, N. Ostrowksy, N. Phys. Rev. Lett. 57, 17 (1986).

13. G. Fytas, S.H. Anastasiadis, R. Seghrouchni et al., Science 274, 2041 (1996).

14. M. Polverari M., T.G.M. Vandevent, Langmuir 11, 1870 (1995).

15. E. Eckert, E. Bartsch, Phys. Rev. Lett. 89, 125701 (2002).

16. N. Garnier, N. Ostrowsky, J. Phys. II 1, 1221 (1991).

17. M. Hosoda, K. Sakai, K. Tagaki, Phys. Rev. E 58, 6275 (1998).

18. a) P. Holmqvist, J.K.G. Dhont, P.R. Lang, Phys. Rev. E 74, 021402 (2006); b) P. Holmqvist, J.K.G. Dhont, P.R. Lang, J. Chem. Phys. 126, 044707 (2007).

19. D. Ausserré, H. Hervet, F. Rondelez, Phys. Rev. Lett. 54, 1948 (1985).

20. B.J. Berne, R. Pecora, Dynamic Light Scattering: With applications to Chemistry, Biology and Physics (Dover publications, New York 2000).

21. de Gennes P.G., Macromolecules 13, 1069 (1980).

22. L. Lobry, N. Ostrowsky, Phys. Rev. B 53, 12050 (1996).

23. See, for example, G.H. Koenderink, S. Sacanna, D.G.A.L. Aarts et al., Phys. Rev. E 69, 021804 (2004); T.H. Fan, J.K.G. Dhont, R. Tuinier, Phys. Rev. E 75, 011803 (2007) and references therein.

24. See, for example, T. Sun, R.R. Chance, W.W. Graessley et al., Macromolecules 37, 4304 (2004).

25. H.L. Tu, L. Hong, S.M. Anthony et al., Langmuir 23, 2322 (2007). 